Journal Port Science Research vol:2, No:1, 2019

$\overline{\mathrm{PAPER}} \cdot$ Full original article online Free

\title{
Transformations of critical attention center
}

\author{
Huda Yousif Al-Obeidi
}

Directorate of Baghdad Education Karkh III, Ministry of Education, Baghdad, Iraq Albydhdy95gm@gmail.com

\section{تحولات مركز الاهتمام النقدي \\ هدى يوسف العبيـدي \\ وزارة التربية، مديريـة تربية بغداد الكرج الثالثة، بغداد، العراق \\ Albydhdy95gm@gmail.com}

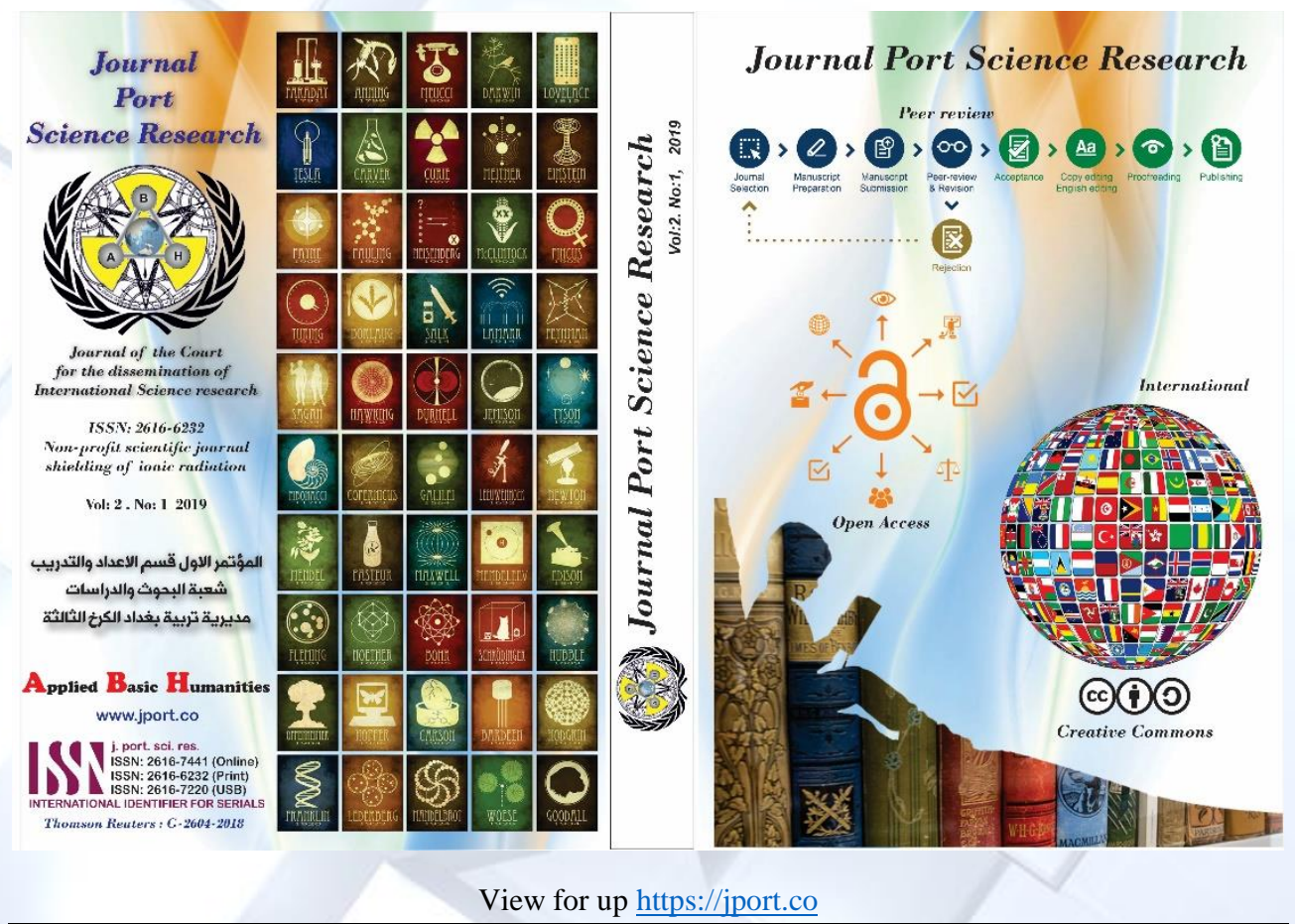

To cite this article: Published 2019 • (C) 2019 Bryant University United States of America Publishing Ltd. 
Ministry of Education Iraqi Directorate of Education Baghdad Karkh III

First Conference Department of Preparation and Training Division of Research and Studies

وزارة التريية العراقية مديرية تريية بغداد الكرخ الثالثة المؤتمر الاول قسم الاعداد والتلدريب شعبة البحوث واللدراسات

\title{
Transformations of critical attention center
}

\author{
Huda Yousif Al-Obeidi \\ Directorate of Baghdad Education Karkh III, Ministry of Education, Baghdad, Iraq \\ Albydhdy95gm@gmail.com
}

\begin{abstract}
The study revolves around what critics and scholars have known as (critical authority), and we start by stating our view of the term. We took the term "critical focus of the curriculum" as an alternative. The critical focus of critic's interest varies according to each approach. As we study this aspect, we must present the critical approaches and their practical movement, and we show our topic according to the emergence and effectiveness of those approaches.
\end{abstract}

Keywords: Stereotyping, literary, criticism.

\section{تحولات مركز الاهتمام النقلدي}

\author{
هلدى يوسف العبيدي \\ وزارة التربية، مديرية تربية بغداد الكرج الثالثة، بغداد، العراق \\ Albydhdy95gm@gmail.com
}

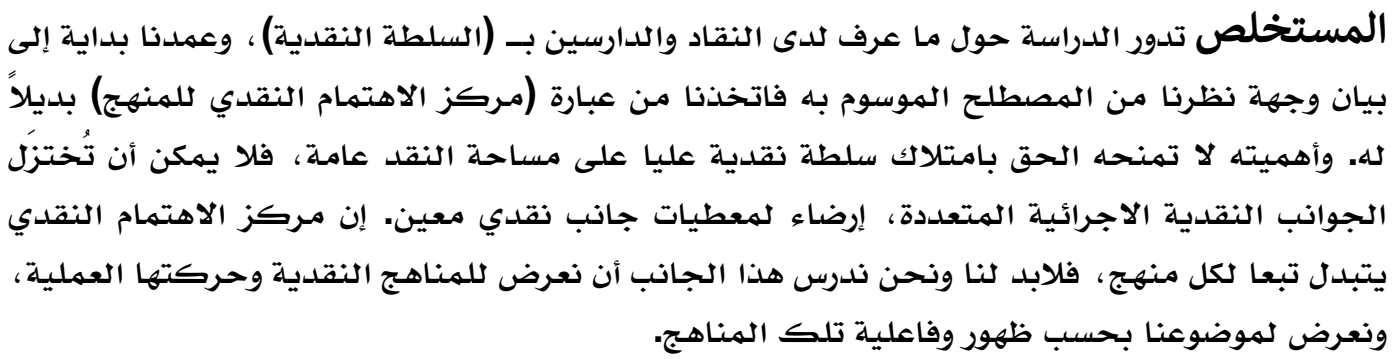


يتناول البحث إثكالية معروفة في حقل الدراسات الاشتغالان النقدية وهي مشكلة تحديد ما يدعى بالسلطة النقدية وما يتعلق بهذا الجانب من مسببات وما يترثح عنه ، فضلا عن الهصطلح الموسوم به والذي نعدّه فيما يعده أحد أوجـه المشكلة. أهمية البحث

يتمحور الموضوع الذي نحن بصدده، حول ما يعده النقاد، جانباً مهماً ، أو أحد الجوانب الأهم في العملية النقدية، فيما يعده البعض منهم مـ تسليههم بتلك الأهمية بأنه الهظهر الأكثر إشكالية وجدلا في الخطاب النقدي العربي [1]، وهو ما يصطلح عليه بسلطة المنهج النقدي، أو السلطة النقدية للمنهج. والمشكلة التي طالما اشتد النقاش حولها في هذا الأمر هي في تحديد الجانب النقدي الذي له الحقى في تسنهم السلطة النقدية، دون منازع، وهذا بطبيعة الحال غير مهكن لأسباب ستتبين لنا خلال خطوات البـحث.

\section{• أهداف البحث وفرضياته}

و يهدف البحث إلى المساهمـة ضمن الجهود التي تسعى لكسر القوالب الجامدة في التعاطي مع موضوعة

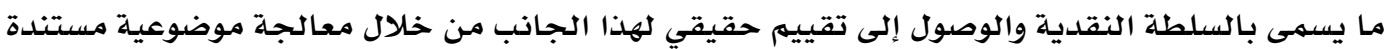

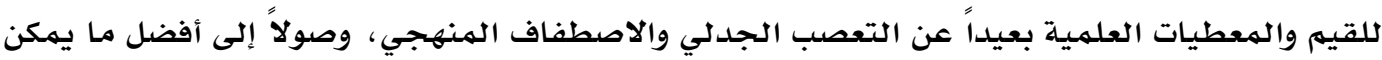
الوصول إليه في حل إحدى الإثكاليات الشائعة في الساحة النقدية، ومن دون أن ندخر أو نتجاهل مستويات اخرى فيها تتمثل في تهركز هذه السلطة، فضلا عن المصطلح الموسوم به، واللذين نفترض بطلانهما ، وسنسعى لإيضاح رؤيتنا ورأينا بصددهما بالحلول التي سيصل اليها البحث بشأنهما [2].

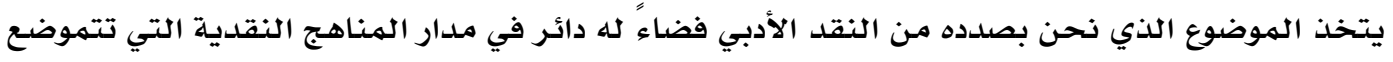
فيها ثيمة [3] البحث التي نحن بصددها، ويستهدف البحث ثلاث جنبات من الموضوع وهي: مصطلح

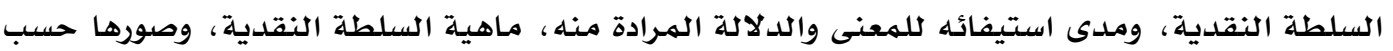
الهناهـ النقدية، تموضـع أو تمركز السلطة النقدية [4] ، حقيقة موضوعية أم تعصب فكري واهم.

\section{• مصطلحات البحث}

يتناول البحث مصطلحات نقدية وأدبية عديدة، لكن المصطلحات الرئيسية ذات الارتباط الموضوعي

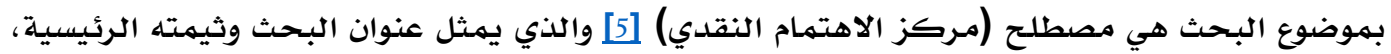

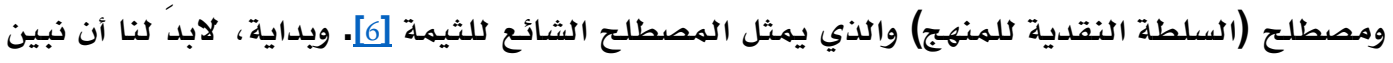
موقفنا من المصطلح الذي نرى فيه الكثير من المغالطة لأسباب تتعلق في جانب منها بالهعنى اللغوي لمفردة السلطة، والمفارقة الحية على مستوى الدلالة للمفردة، فيما تتعلق في جانب آخر منها بالفعالية

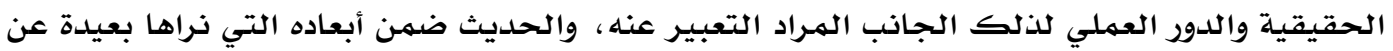
صياغة ذلك المصطلح الذي آمل من المختصين أن يتحروا بديلاً أقرب للمعنى وأوسـع استيفاء لمتطلبات ذلك الجانب النقدي. ولعل عبارة (مركز الاهتمام النقدي) التي تضمنها العنوان الذي وسمنا بـه موضوعنا هذا، هي العبارة الأنسب للاصطلاح على الجانب النقدي المعبر عنه بالمصطلح الشائع (سلطة الهنهج

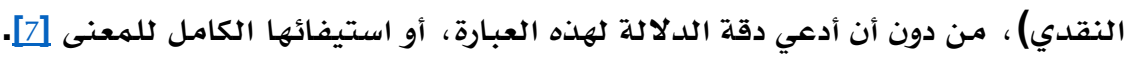

العبيدي، هدى يوسف. (2019). تحولات مركز الاهتمام النقدي. Journal Port Science Research، 2(1) ، 10. 


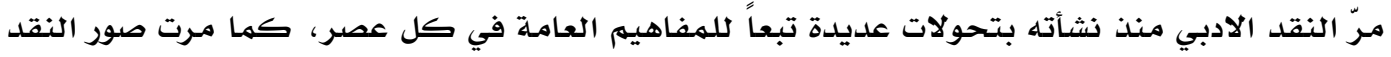

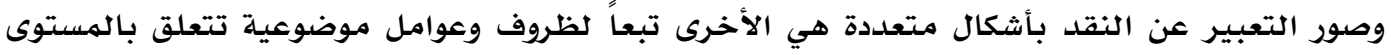

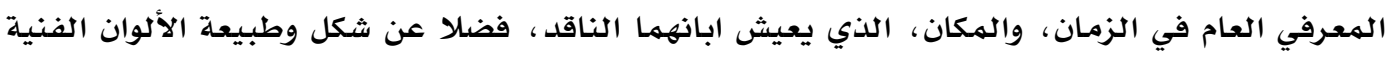

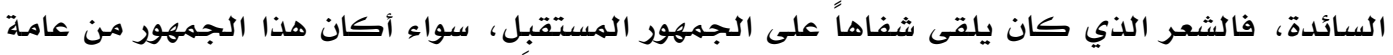

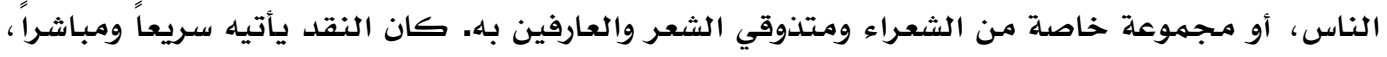

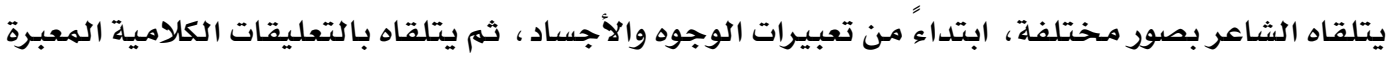

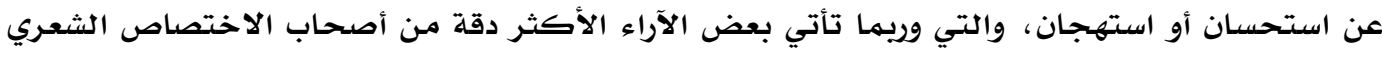

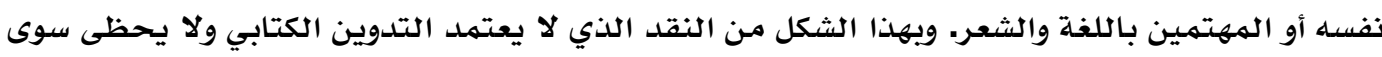

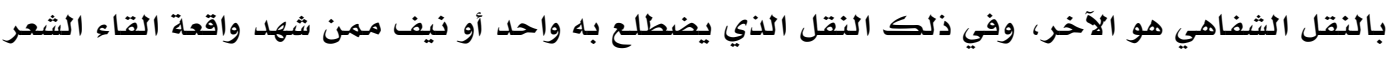

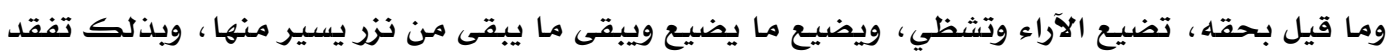

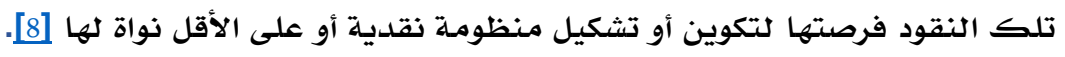

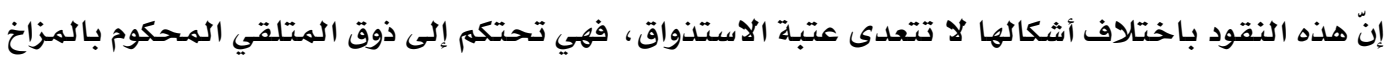

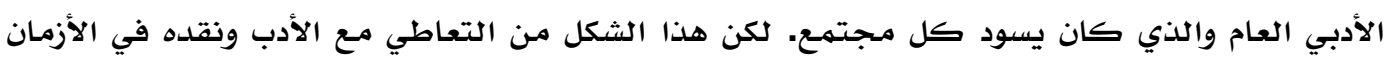

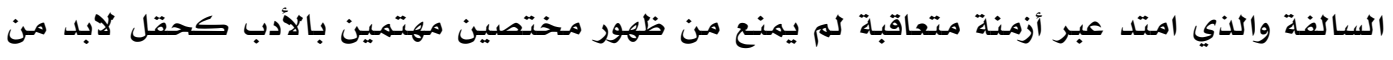

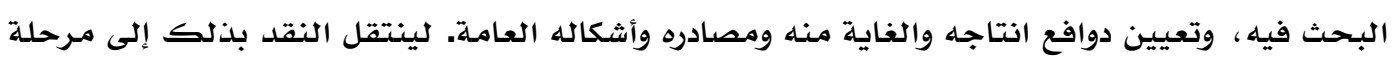

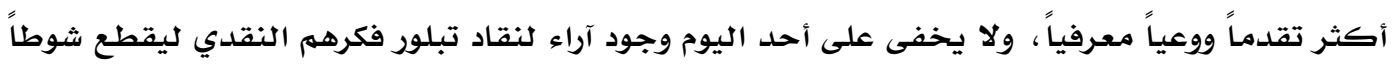

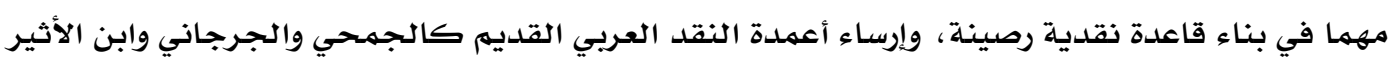

$$
\text { والقرطاجني وسواهم [9]. }
$$

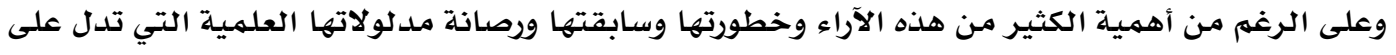

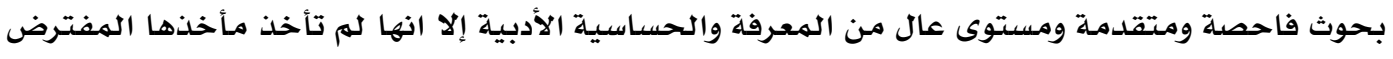

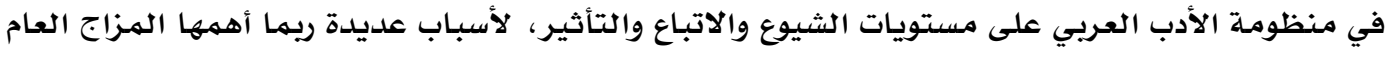

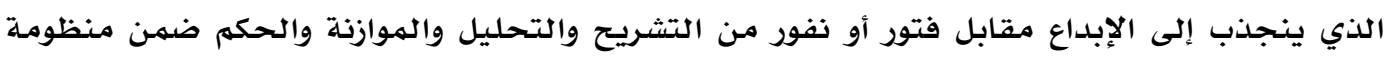

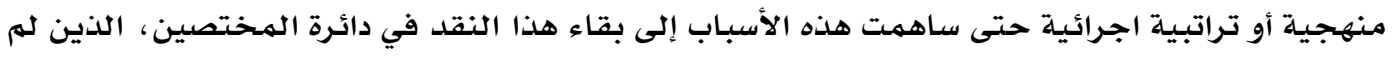
يضف تابعيهم على نتاجهم ولم يساهموا بتغيير أو تطوير.

عائشة حويشي (2015): نقد الشعري لمحمد مندور (دراسة وصفية تحليلية) كان مفاد الدراسة إن النقد

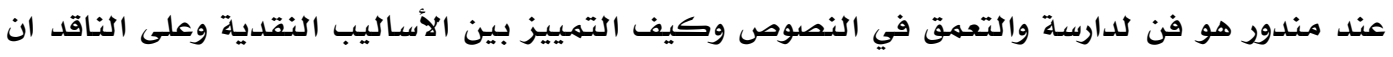

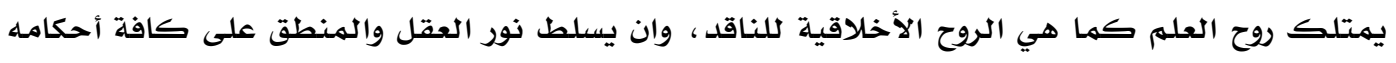

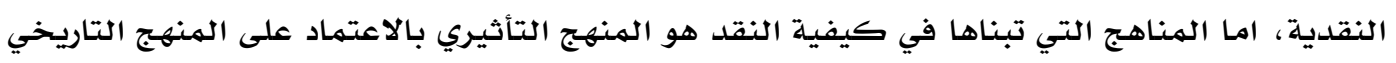

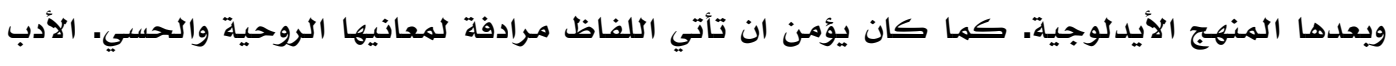

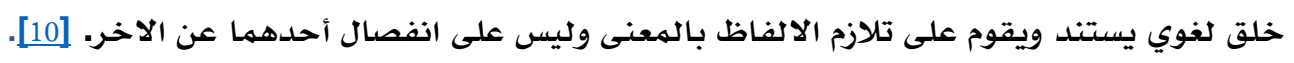
هوارية خوالف (2016): "دراسة وصفية تحليلية لكتاب التفكير النقدي عند العرب لعلي عيسى العاكوب"

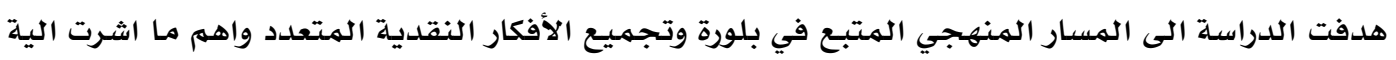

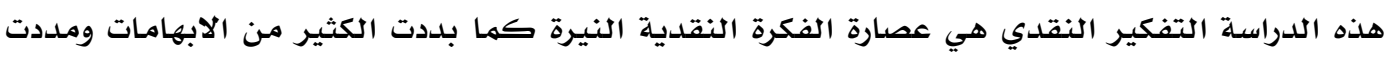

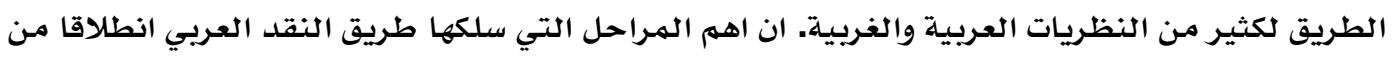

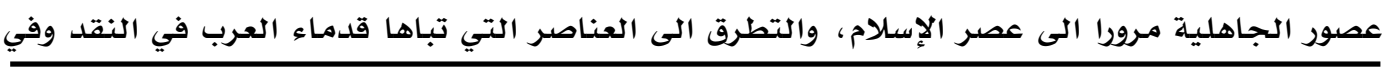


تشكيلة وبناء الشعر، نظرة على قضية السرقات الأدبية والشعرية على أنها احتذاء واستعانة، تصور ونظرة

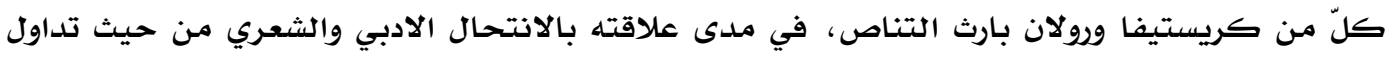

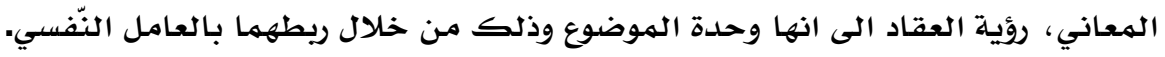

\section{2. المنهجية والإجراءات}

المنهجية هي مجموعة الأفكار والأساليب التي نستخدمها في القراءة العملية للأدب. من خلال المنهجية والإجراءات الأدبية، لا نشير إلى معنى عمل الأدب، بل إلى المنهجية التي تكشف معنى الأدب. النظرية الأدبية هي وصف للمبادئ الأساسية، يمكن للمرء أن يقول الأدوات التي نحاول من خلالها فهم الأدب. تعتمد جميع التفسيرات الأدبية على أساس نظري، ولكنها يمكن أن تكون بمثابة مبرر لأنواع مختلفة جداً

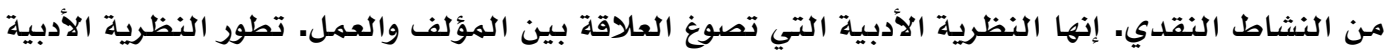
أهمية العرق، والطبقة، والجنس في الدراسة الأدبية، سواء من وجهة نظر سيرة المؤلف وتحليلها لوجودها الموضوعي ضمن النصوص. تقدم النظرية الأدبية مناهج متباينة لفهم دور السياق التاريخي في التفسير وكذلك أهمية العناصر اللغوية وغير الواعية للنص. يتتبع علماء النظريات الأدبية تاريخ وتطور الأنواع المختلفة - السردية والدرامية والغنائية - بالإضافة إلى ظهور الرواية والقصة القصيرة في الآونة الأخيرة،

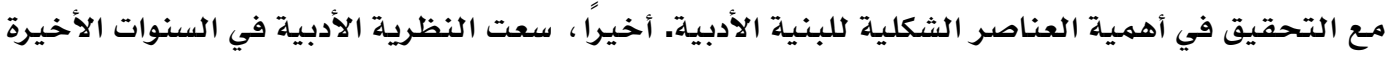

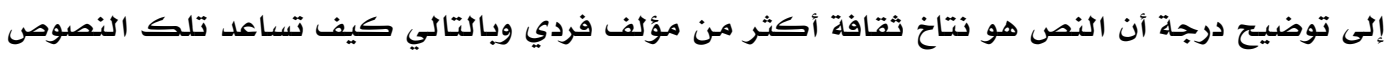
على خلق الثقافة. قصيدة غنائية - بالإضافة إلى ظهور أحدث للقصة والرواية القصيرة، مع التحقيق في التحني أهمية العناصر الشكلية للبنية الأدبية. [11]، بانتقاله نوعية على مستوى الزمان والمكان، نرسو بعدها

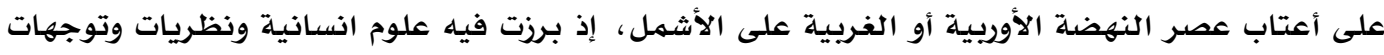

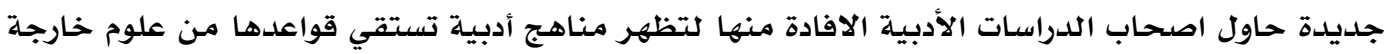
عن الأدب من جهة ومن جهة هي تبحث في الجوانب التي تختص فيها تلك العلوم، فالنقد الاجتماعي مثلا يبحث في الجوانب الاجتماعية للأديب وتأثيرها عليه ويحاول أن يربط بين تلك الجوان الجوانب الاجتماعية للمؤلف وبين النص، وهذا غالباً لا يغني النص ولا العملية النقدية، وكذا الحال في المنهج التاريخي الذي كثيراً ما يتوجه أصحابه بنا نحو البيئة والعصر والمدرسة التي نشأ فيها الشاعر ويحاولون أن يقنعوا

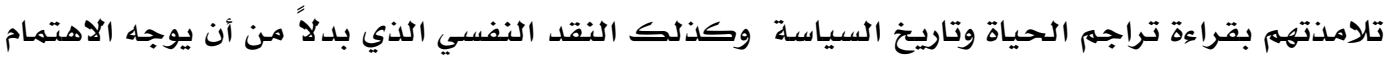

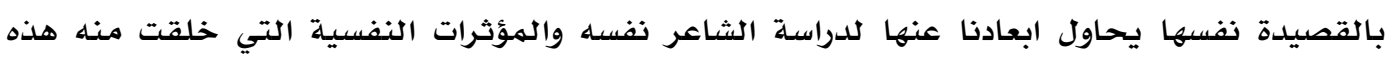
الشخصية أو تلك وجعلته يسلك هذا السلوك أو ذاك [12].

ثم ظهر الاتجاه الذي ينظر الى أن معالجة النصوص لابد أن تنطلق من الذات الأدبي أي من النصوص نفسها أي إن الرؤية النقدية يجب أن تتوجه الى النص وتنطلق من معطياته ليظهر الاتجاه النصي والذي بدأ بتحري الجوانب الفنية والجمالية والشكلية داخل النص، ويعود هذا الاتجاه النقدي إلى ظهور تجمعين

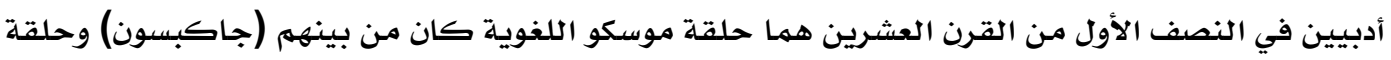
بطرسبورغ ، عملت هاتان الجماعتان على تأسيس تقاليد حوار واثارة جدل مهم بنّاء عكس ازدواجية اهتماماتهم الألسنية والجمالية معاً، وجعلوا الآثار الأدبية محور النشاط ومركز الاهتمام النقدي في

العبيدي، هدى يوسف. (2019). تحولات مركز الاهتمام النقدي. Journal Port Science Research، $2(1)$ ، 10. 
مقابل اغفال ما يتصل بها من عوامل ومرجعيات، ساعين إلى خلق علم أدبي مستقل ينطلق من جوهرية

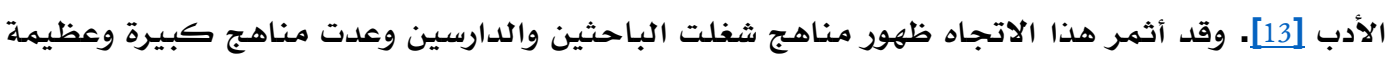

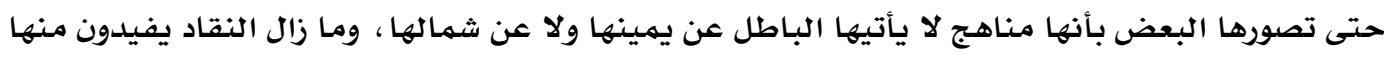
ويعملون حسب معطياتها ولعل أبرز هذه المناهج هو المنهج البنيوي والأسلوبي والسيمولوجي، والتي ترى التها أن الحقيقة الجوهرية للنص تكمن في البنية الداخلية له. بعد أن أخذت البنيوية والمناهج النصية

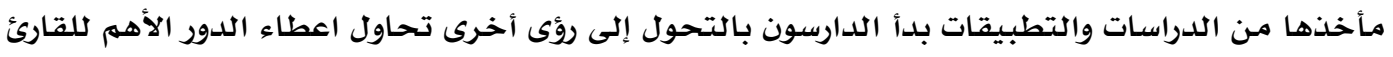

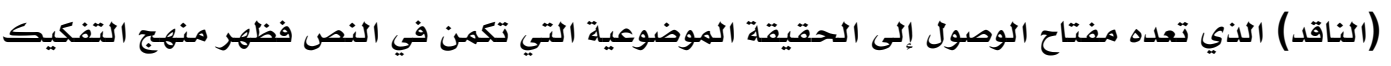

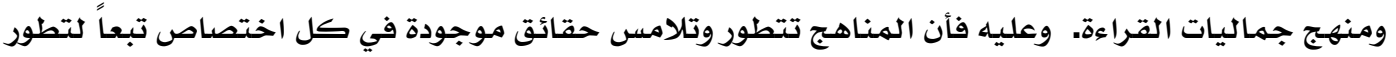
ذلك الاختصاص والمناهج الادبية تطورت وفق هذا المفهوم. وفي المناهج النقدية الوجه الأكثر حضوراً

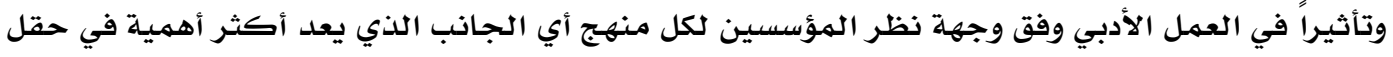

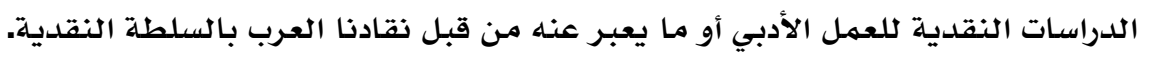

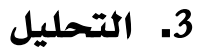

إنّ مركز الاهتمام النقدي ليس شكلاً ثابتاً أو جانباً معيناً يتكرر في كل اتجاه نقدي بل ان هذا تحليل المركز جانب من جوانب العمل الأدبي وتارة هو أحد جوانب تكوينه، فيما يكون أحد عناصر العملية النقدية تارة أخرى. وبشكل عام فمركز الاهتمام النقدي هو الجانب المعبر عن روح وعماد الادي التهاد المنهج.

\section{مركز الاهتمام النقدي حسب المناهج النقدية}

أولا: المذهب الانطباعي يكون مركز الاهتمام به هو القارئ (الناقد) ورؤيته الخاصة للنص الأدبي، وتشترك

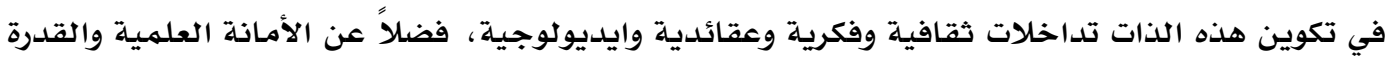
على الحيادية، لتشترك بمجموعها في طبيعة وشكل نظرتها ومعالجتها للأعمال الادبية، وبكل الأحوال

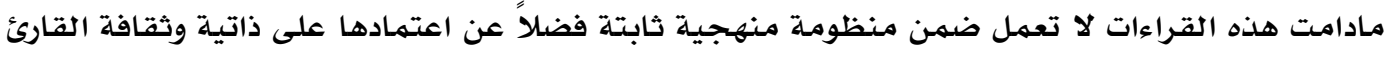
المعرضة للمؤثرات الخارجية العديدة فأنها تبقى في أصلها قراءات غير محتكمه إلى منهج واضح المعالماته

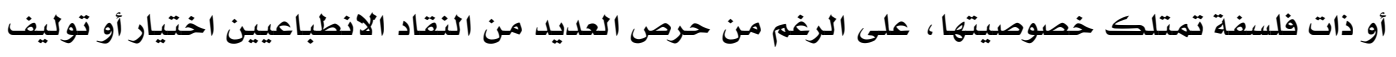

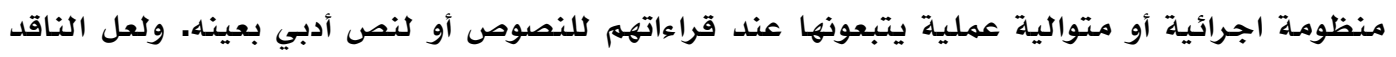
الدكتور علي جواد الطاهر خير من يمثل ذلك النموذخ فهو يمنهج لنفسه ويضع القواعد العامة للعملية

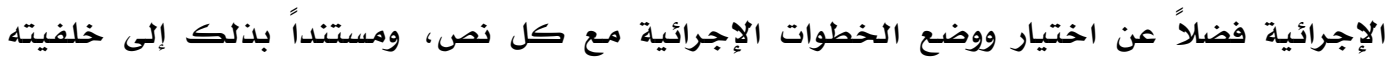

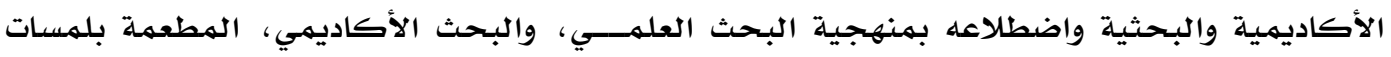
نقدية [14].

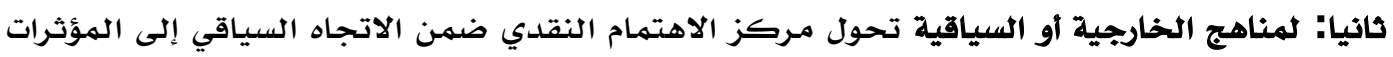

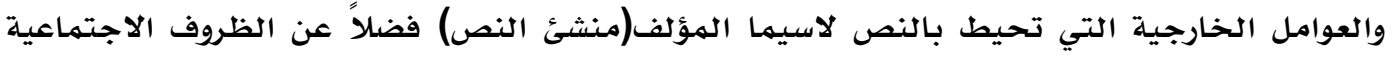

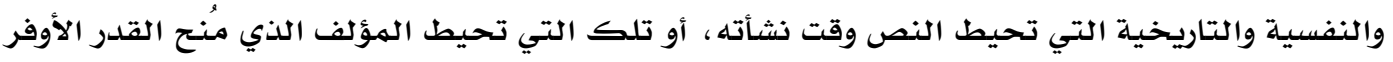

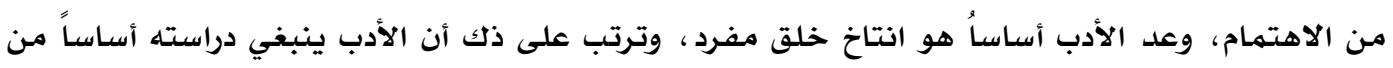

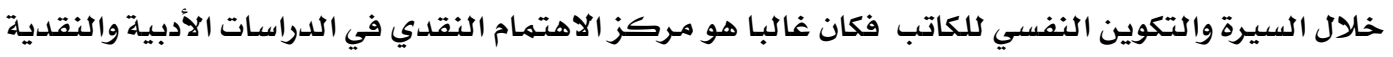


وعدّه اصحاب هذا الاتجاه هو العامل الأهم والأسساسي في العمل الأدبي. وهناك فريق آخر بحث عن العوامل الأساسية التي تحدد الخلق الأدبي في حياة الإنسان العامة في الظروف الاقتصادية والاجتهاعية

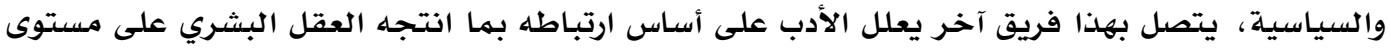

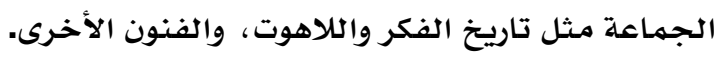

ثالثا: بين الانطباعية والمناهج السياقية إن الاتجاه الانطباعي في نقد الأدب ونقد كل مظاهر الحياة والمعرفة موجود منذ الأزل، وقد تفاوتت درجات شيوعه واتباعه في أزمان ومدد مختلفة، وإلى يومنا هذا

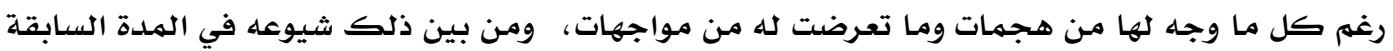
والمدة الكلاحقة لظهور المناهـج السياقية ، فبعد أن أخذت المناهـج السياقية مـأخذها في الساحات النقدية، تعرضت هي الأخرى إلى ردود أفعال قوية في الوقت نفسـه من لدن الانطباعيين الذين عاد مدهبهم للشيوع في هذه المدة، وفي كل الأحوال ففي رأينا أن الانطباعية هي مدهب أو تيار نقدي وليس منهجا بالمعنى الدال للكلمة وليس ثمـة مسوغ للاصطلاح عليه بالمنهج. وعلى أية حال فبالعودة لمعركتهم أي الانطباعيون نرى أن ردود الأفعال على السياقيين اشترك فيها جماعات الاتجاه النصي الذي ظهروا على الى

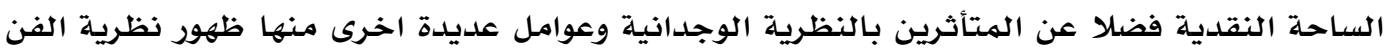
للفن مـا صنـع مواجهة مباشرة بين ما نادت به تلك النظرية من العزلكة الجمالية وانطواء الفن الجميل

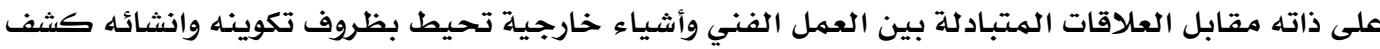

عنها السياقيون [15].

وتلك العلامات والاشياء الخارجية هي ما أولته هذه المناهـج حقل الاهتمام النقدي كما أشرنا تاركة النص وايحاءاته وتشكيله الفني وما يتوفر عليه من تراكيب وجماليات بنائية إلى ذوق وثقافة المتلقي الذي له يكن هو الآخر قد توفر لديه ما يثير حساسيته الأدبية نحو هذه العناصر والقيم الفنية إلا بعد ظهور الاتجاه النصي. رابعا: الاتجاه النصي واللسانيات بعد هذه المخاضات في انطباعات القارئ والمؤثرات الخارجية وشخصية

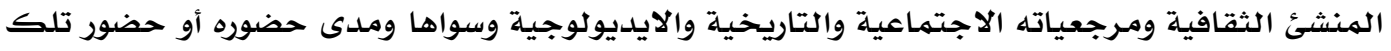

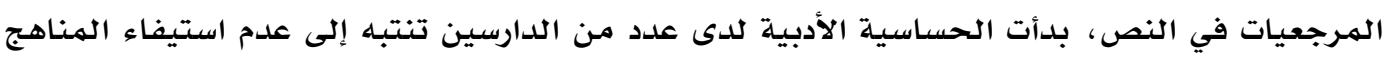
التي تعتمد هذه الجوانب في معالجاتها للنصوص لحق النص وأهميتته وأهمية العملية الأدبية برمتها فضلا عن الشعور بضياع أهمية مكونات النص وشكله البنائي ورسائله المكنونة بين سطوره والعلاقات بين تلك العناصر وأهمية شكل تلك العلاقات في المؤديات التي يقصدها النص فصار أن ظهرت مجموعتي الشكلانيين الروس في بداية القرن العشرين كها أشرنا في بدية البحث كما اعقبت هاتان الجماعتان

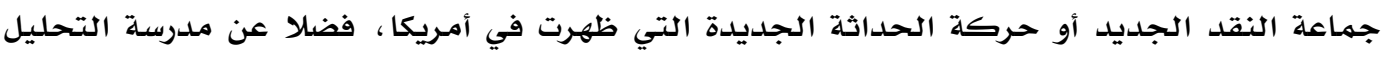
اللفظي في انكلترا والتي عضدت تلك الآراء والتوجهات على الرغهم من تعدد الاتجاهات الأدبية التي التي ينتمون اليها وينطلقون منها كاتجاه التحليل اللفظي الذي ينتهي اليه (روبرت جريفر) والاتجاه

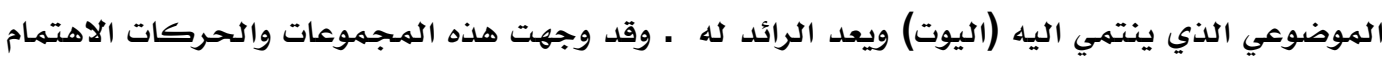

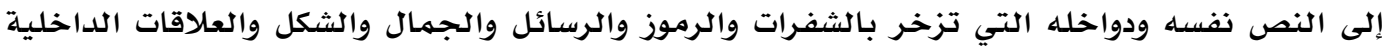

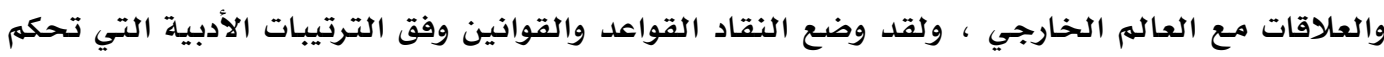
النصوص بصورة عامة حتى تشكلت المفاهيم النصية، فظهرت الدراسات اللسانية التي أنتجت قواعد ونظريات تطورت الى مناهج نقدية علمية تبحث بالنص وتتحرى تراكيبه وبناءاته وجماله والجوانب الفنية فيه فكان أن ظهرت وفق هذا الاتجاه مناهج وتيارات (كالبنيوية والأسلوبية والسميولوجية) و الحقيقة الجوهرية للنص الأدبي تكمن في نظر المناهج الجديدة في الضروف المحيطة بالنص بقدر ما تكمن في

العبيدي، هدى يوسف. (2019). تحولات مركز الاهتمام النقدي. Journal Port Science Research، $2(1)$ ، 10.10. 
البنية الداخلية له، تلك البنية التي تشكل عالما مغلقاً لا سبيل إلى الدخول إليه والكشف عن قوانينه، الدالئه

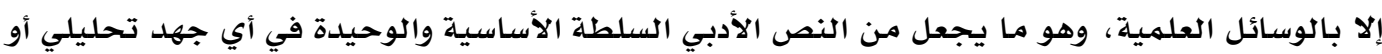

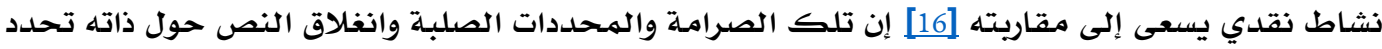
حركية العملية النقدية وتعاطيها مع النص، وتجعل من النص سلطة علوية لا يمكن النظر إليها إلا من

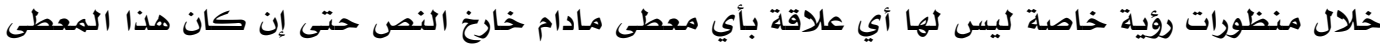

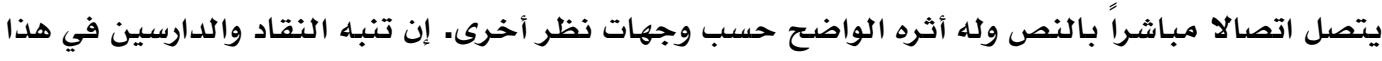

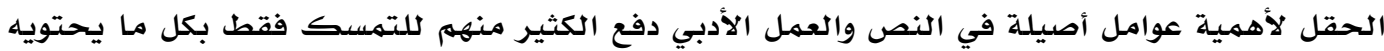

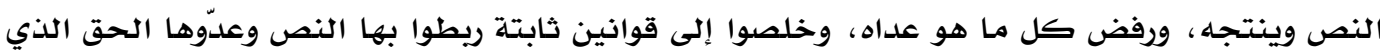

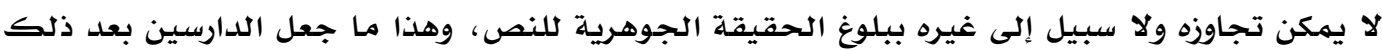

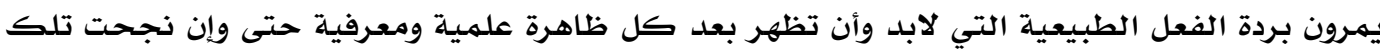

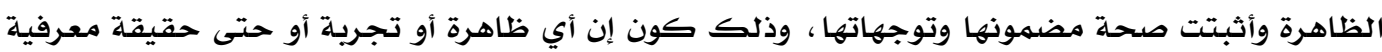

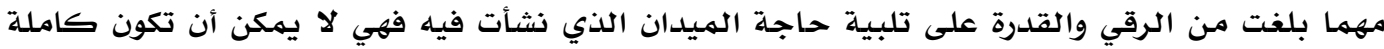

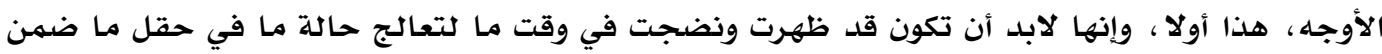

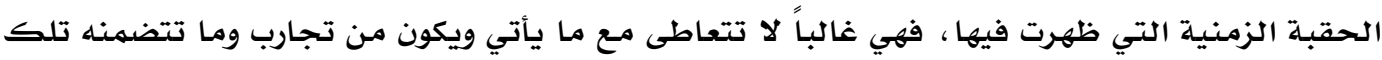

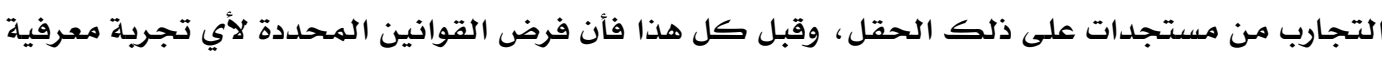

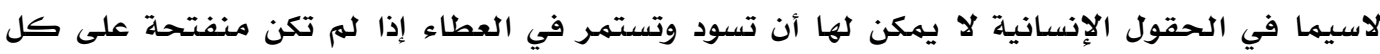

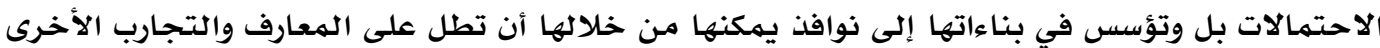

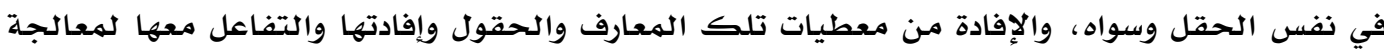
مختلف القضايا الإنسانية والمعرفية.

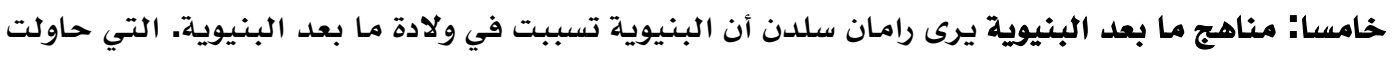

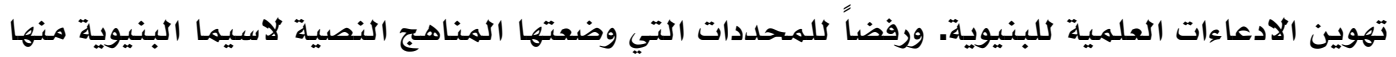

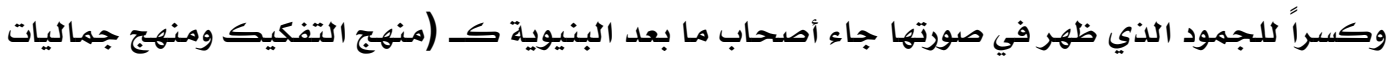

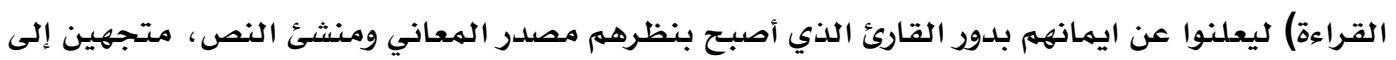

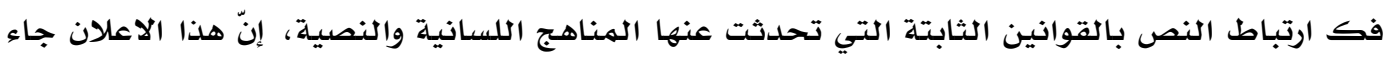

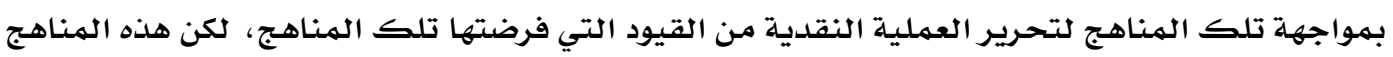

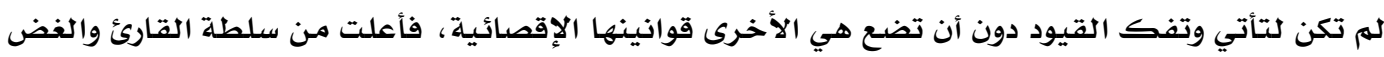

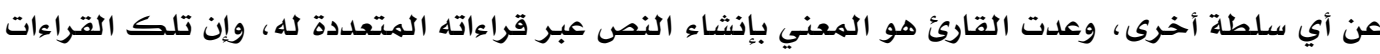

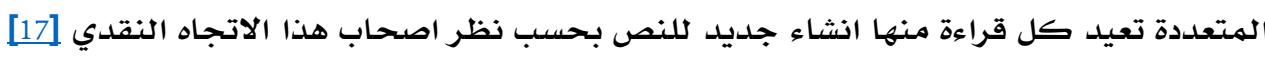

الاهتمام النقدي الصائب بين الانغلاق على المنهج الواحد أو الانفتاح على المعطيات المثمرة في المناهج النقدية الواحت

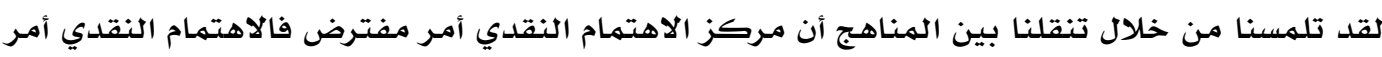

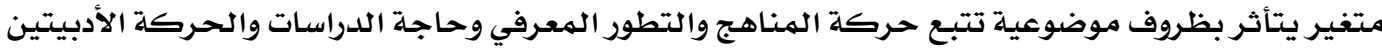

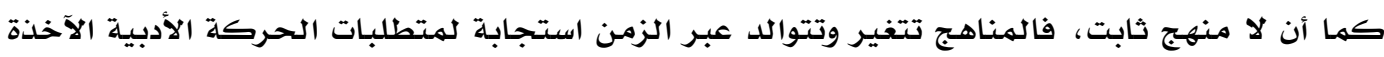

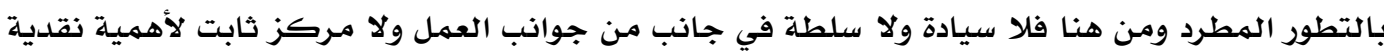

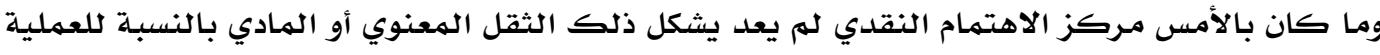

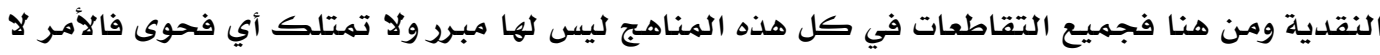


يعود إلى معطى معين بقدر ما يعود إلى نظرة متوازنة تحكم نوع العلاقة بين المناهج ومراكز تأثيرها في

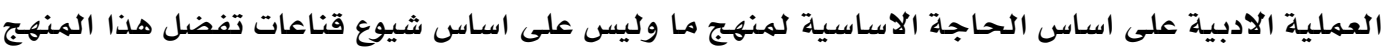

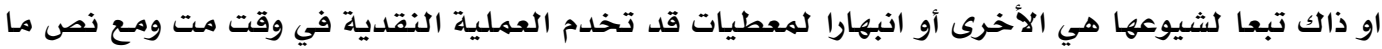

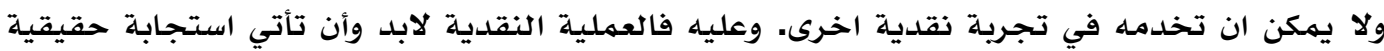

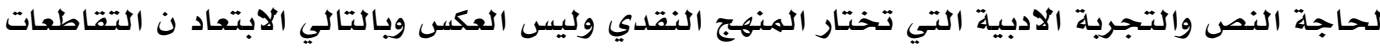

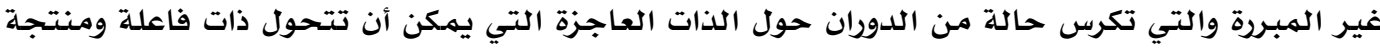

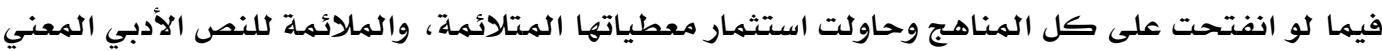

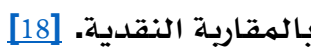

حركية المناهج وأهمية الخطاب النقدي: يقترن ظهور أي منهج بالمستوى المعرفي العام الذي تصل اليه اليه

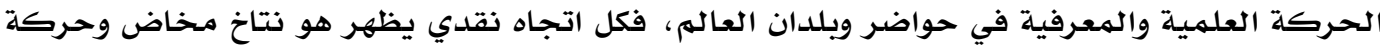

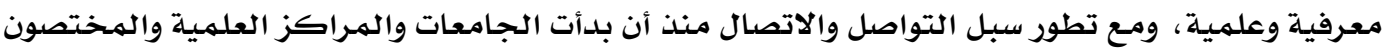

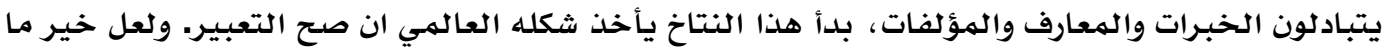

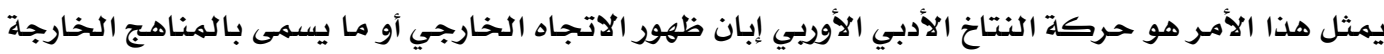

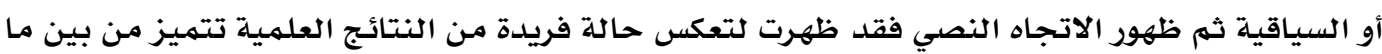

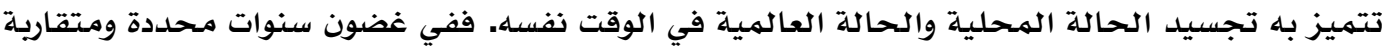

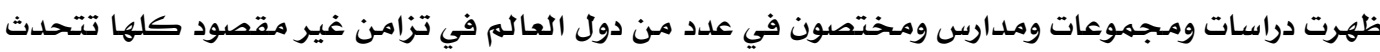

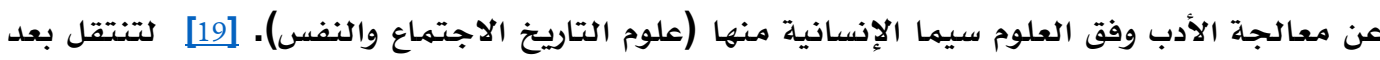

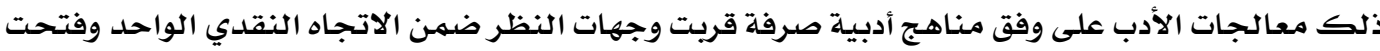

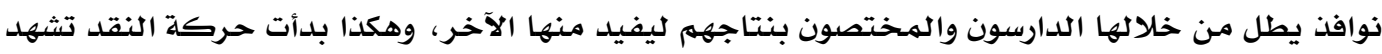

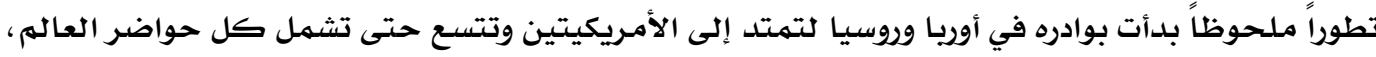

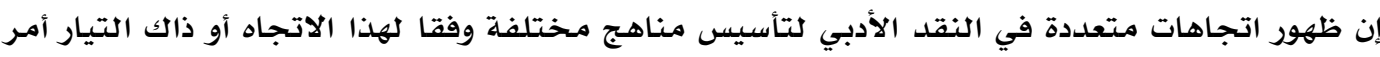

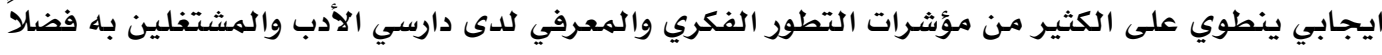

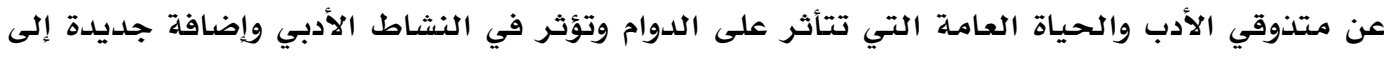

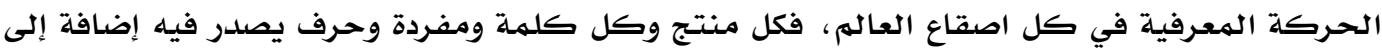

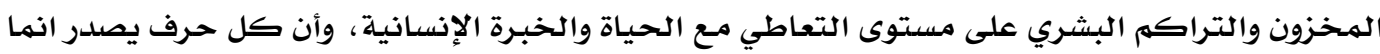

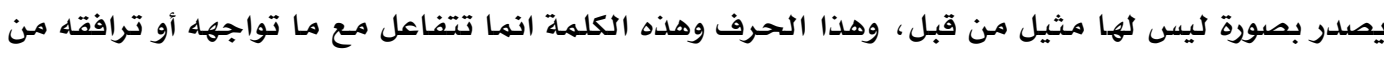

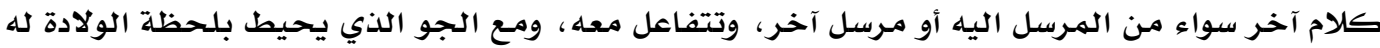

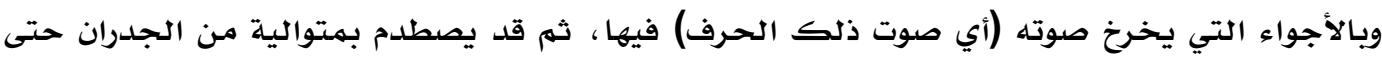

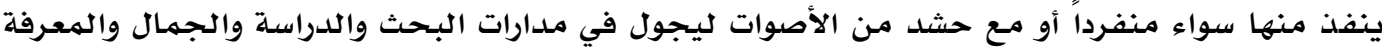

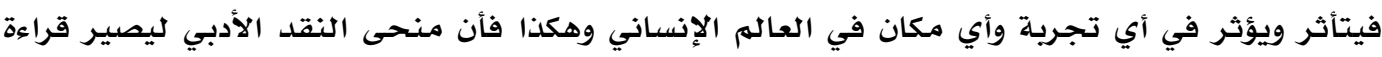

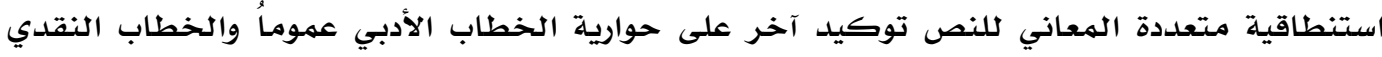

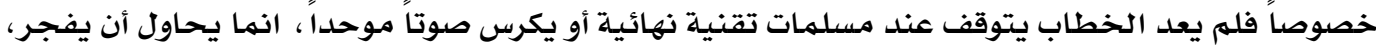

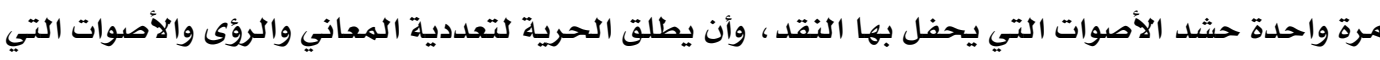

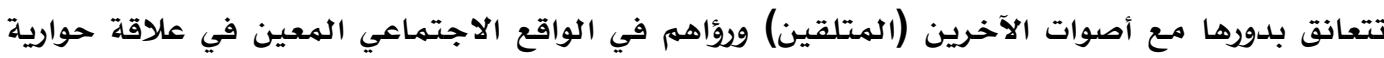




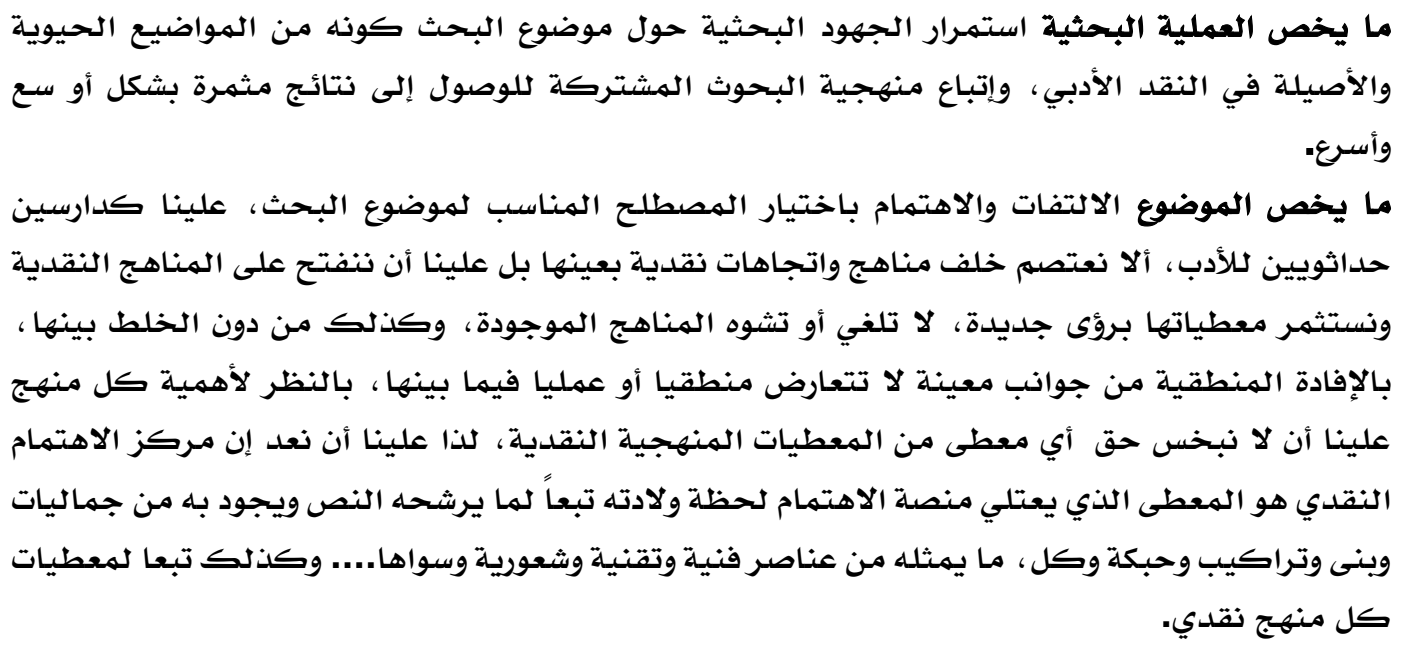

\section{References}

[1] Heidi, S. (2007). لعبة النص: مقاريات نقدية في الشعر والسرد>> > p. 24, Sharjah, Department of Culture and Information. http://altibrah.ae/book/4958

[2] Archive for the Forum > الفصيح>- 3 (group of authors). Index of books and comparative literature. https://al-maktaba.org/book/31862/1601\#p32

[3] Wolf, S., \& Gearhart, M. (1994). Writing what you read: Narrative assessment as a learning event. Language Arts, 71(6), 425-444.

[4] Childs, P. (2010). The Routledge Dictionary of Literary Terms. The Routledge Dictionary of Literary Terms. Routledge. https:// doi.org/10.4324/9780203462911

[5] Moore, T. (2013). Critical thinking: Seven definitions in search of a concept. Studies in Higher Education, 38(4), 506-522. https:// doi.org/10.1080/03075079.2011.586995

[6] Luke, C., Castell, S., \& Luke, A. (1983). Beyond Criticism: The Authority of the School

Text. Curriculum Inquiry, 13(2), 111-127. https:// doi.org/10.1080/03626784.1983.11075873

[7] Marir, S. Ben Suicki, Y. (2016). النقد البنيوي من البنيوية الشكلية إلى سوسيولوجيا النص> > Faculty of Social and Human Sciences, Oum El bouaghi university. http://bib.univoeb.dz:8080/jspui/handle/123456789/1885

Al-Obeidi, H. (2019). Transformations of critical attention center. Journal Port Science Research, 2(1), 1-10. 
[8] Sayed Qutb. (2003). > Cairo: Dar Al Shorouk. https:// foulabook.com/book/downloading/ 677959278

[9] Abu Zeid, Sami Yusuf. (2013). النقد العربي القديه> > Dar Al - Massira for Printing and Publishing. https:// pmb.univ-saida.dz/bullaopac/index.php?lvl=notice display\&id=3186

[10] Hawichi, A. (2015). > نقد الشعر عند محمد مندور (دراسة وصفية تحليلية) >. (Master Thesis), Faculty of Arts and Languages, University Mohamed Boudiaf - M'sila. http://dspace.univmsila.dz:8080/xmlui/bitstream/handle $/ 123456789 / 10591 / 2015032$.pdf?sequence $=1$ \&isAllo wed $=\mathrm{y}$

[11] Culler, J. (1997). Literary Theory: A Very Short Introduction. Oxford: Oxford University. http:// elibrary.bsu.az/books 400/N 19.pdf

[12] Rogers, R., Malancharuvil-Berkes, E., Mosley, M., Hui, D., \& Joseph, G. O. (2005). Critical Discourse Analysis in Education: A Review of the Literature. Review of Educational Research, 75(3), 365-416. https:/ / doi.org/10.3102/00346543075003365

[13] Heidi, S. (2007). النقد الأدبي الحديث ، قضاياه ومناهجه>. Publications, University of the seventh of April, Libya 1998. https:/ / tinyurl.com/y3awbwna

[14] Hussein, Saleh Zamel. (2015) 2005 > مناهج النقد الأدبي في العراق من Paghdad, Dafaf Publications.https:// bookslibrary.online/ files/bookslibrary.online noo08c10c85cbe86f28b3 28da-28229.rar

[15] Mustafa, super. Ali, Abdul Redha (1989). في النقد الأدبي الحديث منطلقات وتطبيقات > Dar Al Kuttab for Printing and Publishing, University of Mosul. http:/ / mohamedrabeea.net/library/pdf/065c7a2e-a9d6-4095-8024-277e110081d5.pdf

[16] Lai, E. R. (2011). Critical Thinking: A Literature Review Educators. Research Reports, (April), 41. https:// doi.org/10.2307/3069464

[17] Eagleton, T., \& Selden, R. (2007). A Reader's Guide to Contemporary Literary Theory. The Modern Language Review, 81(4), 959. https:// doi.org/10.2307/3729614

[18] Edwards, J. A. (2008). The Transcription of Discourse. In the Handbook of Discourse Analysis (pp. 321-348). wiley. https:// doi.org/10.1002/9780470753460.ch18

[19] Levy, Y., \& Ellis, T. J. (2006). A systems approach to conduct an effective literature review in support of information systems research. Informing Science, 9, 181-211.

[20] Thamer, F. (2016). الصوت الاخر: الجوهر الحواري للخطاب الادبي > Baghdad: House of Public Cultural Affairs. http:/ / www.iraqnla-iq.com/opac/ fullrecr.php?nid=398805\&hl=ara 